\title{
Bonding silicon-on-insulator to glass wafers for integrated bio-electronic circuits
}

\author{
Hyun S. Kim ${ }^{\text {a) }}$ and Robert H. Blick \\ Laboratory for Molecular Scale Engineering, Department of Electrical and Computer Engineering, \\ University of Wisconsin-Madison, Madison, Wisconsin 53706 \\ D. M. Kim and C. B. Eom \\ Department of Materials Science and Engineering, University of Wisconsin-Madison, Madison, \\ Wisconsin 53706
}

(Received 16 April 2004; accepted 28 July 2004)

\begin{abstract}
We report a method for bonding silicon-on-insulator wafers onto glass wafers. After pre-cleaning the wafers by an ozone and ultraviolet exposure, followed by mega-sonic water rinse, the SOI wafers are bonded to glass wafers in a vacuum chamber. This is performed at a temperature of $400{ }^{\circ} \mathrm{C}$ under an applied voltage of $700 \mathrm{~V}$. The interface between the glass and SOI wafer is tested mechanically and inspected by electron beam microscopy. Furthermore, we demonstrate removal of the silicon bulk layer after wafer bonding. The quality of the single crystalline Si thin film on the glass wafers has been verified by four-circle $\mathrm{x}$-ray diffraction and scanning electron microscopy. This process will allow us the integration of thin-film electronics in biological sensor applications. (C) 2004 American Institute of Physics. [DOI: 10.1063/1.1794855]
\end{abstract}

In order to combine integrated electronics and biological structures like cell membranes or proteins, two different technological fields have to be merged. While the material of choice for integrated electronics is silicon and by now silicon-on-insulator (SOI), living matter such as cells are best cultivated on inert materials like glass. The approach we have chosen is to apply wafer bonding to directly combine silicon and glass wafers and thus the strengths of both materials, i.e., electronic circuits and bio-compatibility. In the following we will first discuss bonding SOI and glass, while we will then make use of the bonded wafers realizing a $100 \mathrm{~nm}$ single crystalline layer on glass.

A variety of methods for wafer bonding have been presented during the last few years, anodic bonding, direct/ fusion bonding, and intermediate-layer bonding. ${ }^{1}$ We have chosen anodic bonding, ${ }^{2}$ which is superior for bonding glass to glass, silicon to glass or $\mathrm{SiO}_{2}$ to glass. ${ }^{3}$ Pyrex 7740 is a standard glass wafer for anodic bonding. ${ }^{4}$ While borosilicate glass will work, the expansion coefficients do not match and hence breaking or bowing will most likely occur.

Before performing the bonding step, it has to be ensured that the wafers are extremely clean. Otherwise particles that are trapped between the bonded wafers can substantially reduce the yield or spoil whole wafers. The cleaning steps used are: $\mathrm{H}_{2} \mathrm{SO}_{4}: \mathrm{H}_{2} \mathrm{O}_{2}$ followed by a DI rinse. ${ }^{5,6}$ For bonding the cleanliness is the most important factor for void-free bonds. ${ }^{7}$ The simultaneous application of ozone and UV light is highly effective in removing residual organic compounds and modifying surfaces for better adhesion. We have cleaned the wafer with $0.5 \mathrm{\ell} / \mathrm{min}$ of $\mathrm{O}_{2}$ flow at room temperature for 20 min. Following the ozone and UV cleaning step, a megasonic DI wafer cleaning step is preferrable for effective removal of small particles.

Anodic bonding ${ }^{2}$ depends on charge migration to achieve good adhesion, preferably used for silicon and glass wafers. Pyrex borosilicate glass is a typical material that con-

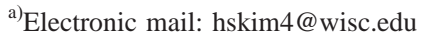

tains sodium oxide $\left(\mathrm{Na}_{2} \mathrm{O}\right)$. The presence of mobile metal ions allows application of a large negative voltage to the glass, attracting and neutralizing the positive ions $\left(\mathrm{Na}^{+}\right)$. This results in a space charge at the glass-silicon interface, producing a strong electrostatic attraction between the silicon and glass wafers, fixing them firmly in place. The mobility of these positive ions is further enhanced by performing anodic bonding at temperatures up to $500{ }^{\circ} \mathrm{C}$. Driven by the electric field, oxygen from the glass is transported to the glasssilicon interface where it combines with silicon to form $\mathrm{SiO}_{2}$, creating the permanent bond field. Figure 1(a) shows the layer sequence of the two wafers bonded.

(a)
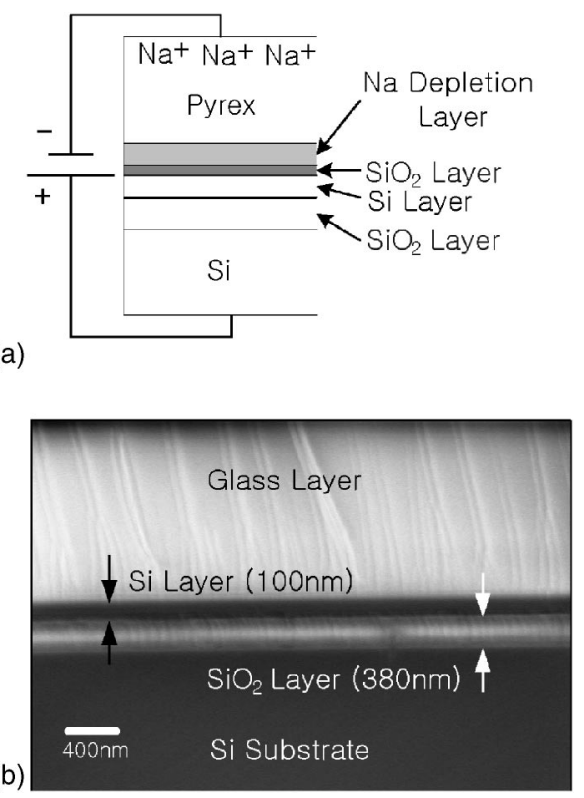

FIG. 1. (a) Schematic representation of the layer sequence. (b) Crosssectional scanning electron microscope image of wafer bonded SOI to glass. 
SOI wafers cannot be anodically bonded through standard methods. The insulator layer in the SOI wafer prevents a current from passing through, which is required for anodic bonding. ${ }^{8}$ A high voltage may cause breakdown of the insulating layer. Nevertheless, it is possible to perform anodic bonding of SOI wafers onto glass by using a high voltage pulse, which will cause a "temporary" breakdown of the insulating layer. In this way SOI wafer bonding becomes possible. The key being that the voltage is high enough for the breakdown of the $\mathrm{SiO}_{2}$ insulator, but not too high to cause permanent damage.

We have successfully bonded 4 in. SOI (100 nm-380 $\mathrm{nm}-510 \mu \mathrm{m})$ and 4 in. Pyrex $(515 \mu \mathrm{m})$ wafers anodically. The bonding experiments were performed in a vacuum chamber at a temperature of $400{ }^{\circ} \mathrm{C}$ with the voltage rising up to 700 $\mathrm{V}$. Ramping to the bonding temperature generally takes 30 min. After this procedure, the sample was cooled down to around room temperature. It has to be noted that the glass must contain a mobile ion, such as $\mathrm{Na}^{+}$. Pyrex 7740 and Schott Borofloat are examples of suitable glasses for anodic bonding. ${ }^{4}$

Visual inspection and destructive tests were performed after bonding the wafers. If fully bonded, the glass is clear, unbonded areas will appear slightly darker, with Newton fringes or circles in the unbonded areas. If the wafers are separated by inserting a razor blade between the wafers, a strong bond will cause fracture of the silicon. The bond interface should be stronger than bulk silicon. If the wafers easily separate without breaking, there was no bonding or only weak bonding. In our case we could not even insert a razor blade and the wafers did not separate at all. In addition we investigated the bonding interface using scanning electron microscopy. The graph obtained gives a cross-sectional view of the SOI-glass interface, shown in Fig. 1(b). As seen the glass wafer is perfectly bonded to the SOI wafer with a well-defined insulating layer.

Apart from bonding the two wafers the next step for applications especially in bio-circuits is to lift-off the bulk silicon substrate, leaving behind a crystalline silicon layer. With a thickness of only $100 \mathrm{~nm}$ the material obtained is perfectly suited for realizing thin film electronic components in bio-sensor circuits. We realized such thin silicon films of $100 \mathrm{~nm}$ on glass wafers by performing first the wafer bonding, followed by wet etch steps. Figure 2 gives a schematic overview of the whole procedure: first SOI and glass are anodically bonded, then the silicon substrate is removed by $\mathrm{KOH}$ wet-etching. Although the thin silicon film is attacked at the edges during $\mathrm{KOH}$ etching, the bonded layers are in an almost stress-free state during $\mathrm{KOH}$ etching step. This effectively reduces the possibility of $\mathrm{KOH}$ penetration into the bonding interface. ${ }^{1}$ In the first etch step the $\mathrm{SiO}_{2}$ layer serves as an effective $\mathrm{KOH}$ etch-stop, protecting the $100 \mathrm{~nm}$ silicon layer on the glass. During the second etch-step using HF the $380 \mathrm{~nm} \mathrm{SiO}{ }_{2}$ layer is eventually removed. A cross-sectional image taken with a scanning electron microscope of the obtained structure is shown in Fig. 3(a). From Fig. 3(a), the single-crystalline silicon film appears to have been uniformly transferred onto the glass wafer. Figure 3(b) shows photographs of the optically transparent structures after removal of the silicon substrate and the $\mathrm{SiO}_{2}$ layer.

The crystalline quality and strain state of Si layer was determined using four-circle $\mathrm{x}$-ray diffraction with twodimensional area detector. Figure 4 shows a two-dimensional Downloaded 18 Dec 2006 to 128.104.198.71. Redistribution subject

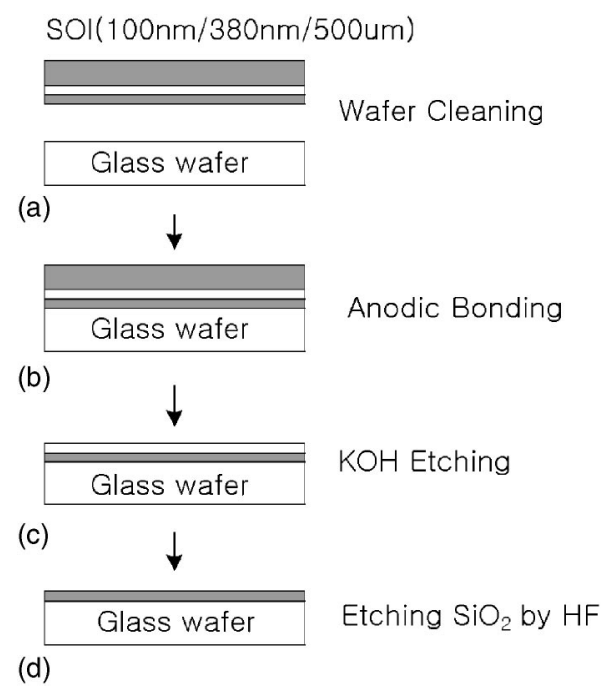

FIG. 2. Process flow of fabricating a silicon layer onto a glass wafer bonding and back-etching: (a) wafer preparation with cleaning steps $\left(\mathrm{H}_{2} \mathrm{SO}_{4}: \mathrm{H}_{2} \mathrm{O}_{2}\right)$ followed by a DI rinse, ozone and UV cleaning, and megasonic DI wafer cleaning. (b) Bonding SOI to glass with an applied voltage $700 \mathrm{~V}$ at a temperature $400{ }^{\circ} \mathrm{C}$. (c) Back-etching with $\mathrm{KOH}$ using the $\mathrm{SiO}_{2}$ layer as an etch stop. (d) Transferred silicon layer on a glass substrate.

image of the x-ray diffraction peak and the integrated intensity plot in $\theta-2 \theta$ domain from a $\mathrm{Si}$ thin film layer on amorphous glass substrate. The only peak observed at $2 \theta$ $=68.26^{\circ}$ in the diffraction scan is (004) Si reflection, which clearly shows the thin Si layer is a single crystal. ${ }^{9}$ The outof-plane lattice parameter is determined to be $(5.43 \pm 0.002) \AA$, which is the same as the value of a bulk $\mathrm{Si}$ single crystal. This confirms the single crystalline Si layer is stress free when transferred onto glass substrate.

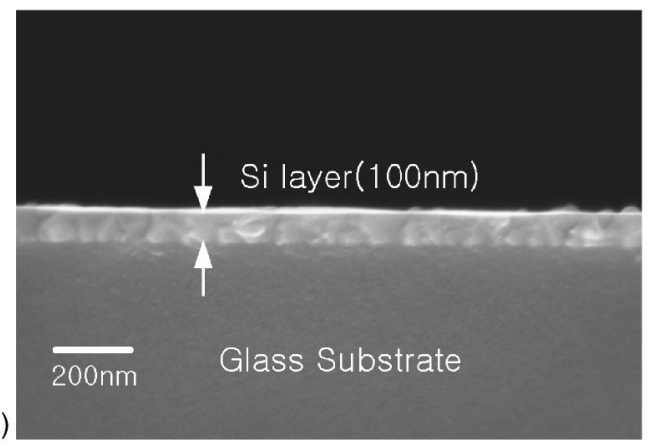

(a)

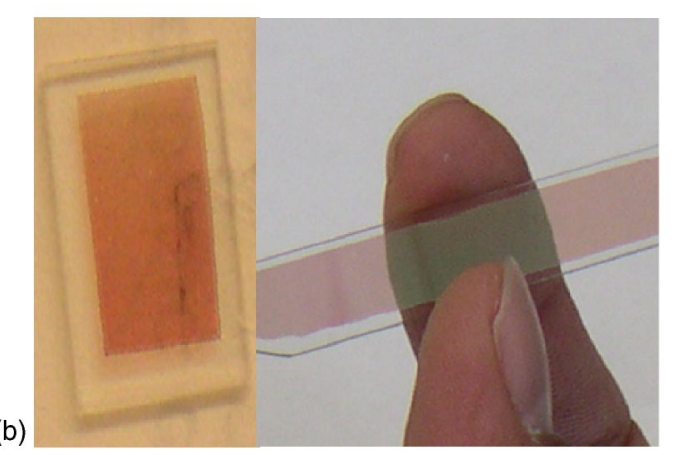

FIG. 3. (a) Cross-sectional scanning electron microscope image of transferred thin silicon layer on a glass substrate. (b) Photographs of the $100 \mathrm{~nm}$

transparent silicon film on a glass substrate. 

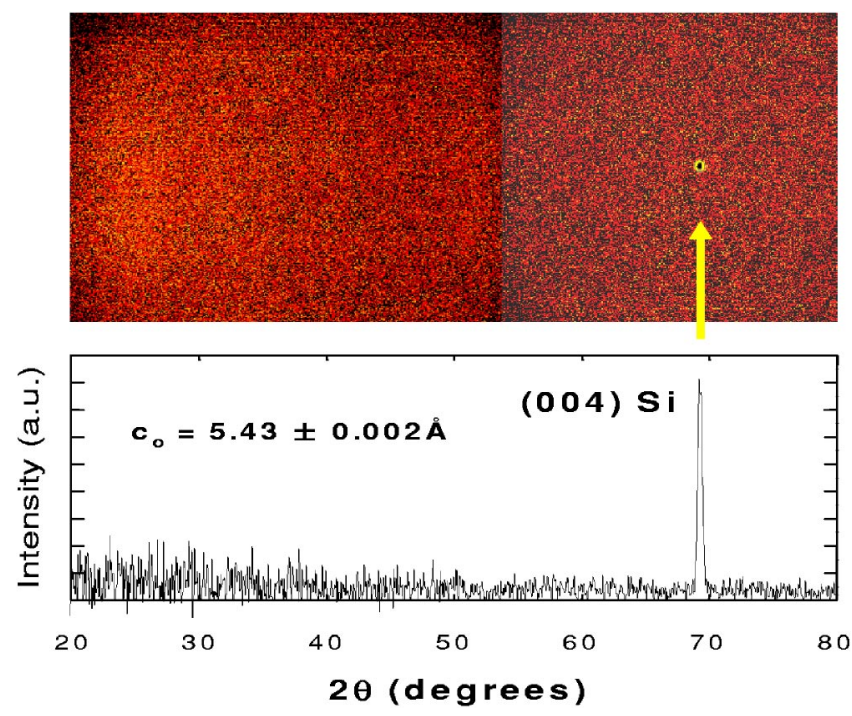

FIG. 4. Two-dimensional diffraction image and the integrated intensity plot in $\theta-2 \theta$ domain from a Si thin film layer on amorphous glass substrate. The lattice constant of Si layer is determined to be $(5.43 \pm 0.002) \AA$.

To summarize, a recipe for anodic bonding of SOI and glass wafers is presented. The surfaces to be bonded are cleaned by a combined ozone and UV cleaning step followed by mega-sonic DI water rinse. Bonding of SOI to glass at an applied voltage of $700 \mathrm{~V}$ was observed at a temperature of $400{ }^{\circ} \mathrm{C}$. The quality of bonding was verified. Removal of the bulk silicon substrate was demonstrated, opening up new avenues for the integration of thin film electronics and inert materials for applications in bio-electronics.

The authors would like to thank Palensky Joshua and the EV Group for their assistance and helpful discussions during this work. This work was supported by the National Science Foundation under Grant No. DMR-0313764.

${ }^{1} \mathrm{Q}$. Tong and U. Gösele, Semiconductor Wafer Bonding, Science and Technology (Wiley, New York, 1999).

${ }^{2}$ E. Obermeier, Proceedings of the Third International Symposium on Semiconductor Wafer Bonding: Science, Technology and Application, 1995, vol. 95-7, p. 212.

${ }^{3}$ M. A. Schmidt, Proc. IEEE 86, 1575 (1998).

${ }^{4}$ M. Harz and W. Brueckner, Proceedings of the Third International Symposium on Semiconductor Wafer Bonding: Science, Technology and Application, 1995, vol. 95-7, p. 315.

${ }^{5}$ K. Ljungberg and A. Söderbärg, Appl. Phys. Lett. 67, 650 (1995).

${ }^{6}$ W. Kern and D. A. Puotinen, RCA Rev. 31, 186 (1970).

${ }^{7}$ R. Stengl, K.-Y. Ahn, and U. Gösele, Jpn. J. Appl. Phys., Part 2 27, L2364 (1988).

${ }^{8}$ J. Haisma, G. A. C. M. Spierings, U. K. P. Biermann, and J. A. Pals, Jpn. J. Appl. Phys., Part 1 28, 1426 (1989).

${ }^{9}$ B. Cullity and S. Stock, Elements of X-ray Diffraction (Prentice-Hall, Englewood Cliffs, NJ, 2001). 